but no substantial advance was made until the occasion of an outbreak at Hendon in 1882, which, having first been investigated by Dr. Cameron, was afterwards also inquired into by Mr. Power. Here, again, nine-tenths of the houses invaded received their milk from one dairy, the houses being those which were best circumstanced from a sanitary point of view. And here it was further noticed that there had been a special incidence of the disease on people, whether children or adults, who habitually consumed uncooked milk in large quantities. The source from which the milk had received its infection was, again, involved in much obscurity, but there were circumstan'es which led to the surmise that the infectiveness had possibly been brought about by causes operating through the cow herself, and enabling her to furnish a milk capable of inducing diphtheria and an allied sore throat in the human subject; and the milk itself gave some physical indications of this But no disease in the cow other than some possible trivial ailment, which, according to veterinary judgment, did not prevent the cow from being regarded as healthy, harl as yet been made out.

So ma ters remained until 1886 , when a very severe diphtheria epidemic appeared amongst the better classes living at York Town and Camberley. The onset was sudden, 88 marked attacks and 5: of lesser severity taking place in three weeks and a half. Of those attacked, 84.2 per cent. received their milk from one dairy, which thus came under suspicion, and during ten days of special severity every one of the 48 houses invaded took the supply in question. And this specialty of incidence quâ milk was quite different from any other incidence that could be made out. Here, again, the amount of milk consumed was seen to have to do with risk of diphtheria. In certain milk epidemics, where other infectious diseases had been (•oncerned, Dr. Thorne had been led to believe that there was a special infectivity attaching to cream which he thought had probably entangled the specific particles of disease, and so carried them to the surface. But this view could not account for all the circumstances noted at Camberley. Indeed, if any additional risk was incurred by cream drinkers, it was altogether overwhelmed by the danger attaching to the storage of milk, the skim milk resulting from the storage being as infective as the cream, and it seemed as if this storing gave opportunity for the cultivation of the specific organisms of disease which did not otherwise occur. Subsequent experiences of Dr. Klein have gone far to confirm this view.

In the meantime diphtheria prevalences which could unmistakably be attributed to milk were increasing, and suspicion had arisen that a number of the lower animals might be concerned in it. The early experiences of the medical department of the Local Govermment Board having been referred to as to this, Dr. George 'Turner's, Dr. Robinson's, and other observations were quoted. The existence of a disease akin to diphtheria was found amongst fowls which had pecked at diphtheria sputa, amongst turkeys from whom the disease had apparently spread to the human subject, and also amongst other animals, notably cats. With regard to cats, the disease had appeared to be transmissible both from them to children, and from the latter back to cats, and a number of occurrences of diphtheria were quoted in this connexion. Noah Webster's writings in 1800 were also recalled as to the occurrence of fatal " angina " in man synchronously with a "pestilence amongst cats," and the more recent labours of the medical department of the Local Government Board, in so far as the special pathology of the disease was concerned, was then entered into in some detail. Latterly Dr. Klein had been experimenting on certain animals with the membrane of diphtheria, and though he had failed as regards birds, it was otherwise with cats, on the cornea and conjunctiva of which animals a disease infective amongst cats could be produced by inoculation from human diphtheria membrane; and from this induced disease bacilli could be isolated resembling the Klebs-Löffler diphtheria bacilli. From this, Dr. Klein was led to study this bacillus more in detail, and he succeeded not only in confirming Löffler's view that the organisms hitherto regarded as one, included a bacillus potent to induce diphtheria and a pseudodiphtheria bacillus, but he also showed that whilst the two forms of organisms were so far alike as to be indistinguishable microscopically and otherwise. they differed in their power of multiplication when cultivated at different temperatures; the true diphtheria bacillus multiplying, in milk, for example, at the ordinary temperature of the air. This discovery had, it was pointed out, important bearings on the special virulence of diphtheria induced as the result of drinking an infective milk which has been stored for purposes of securing cream or otherwise. At this stage Dr. Thorne recalled the fact that in nearly all the carefully recorded cases of milk diphtheria there had been evidence either of some "trivial " ailment in the milch cows, showing itself, for example, on their udders and teats by means of vesicles, "chaps," etc., or some story of a physical alteration in the quality of the milk, such as was shown by a condition of " ropiness," which dairy farmers admitted to be due to some condition affecting the cow herself. And he then gave an account of Dr. Klein's most recent researches as to milch cows-researches which are to be fully recorded in Dr. Buchanan's forthcoming report on the proceedings of the medical department of the Local Government Board. Briefly told they amount to this. Milch cows inoculated with human diphtheria membrane accuire a definite specific disease which may or may not, in the end, be fatal. The milk apparatus of the animal continues to furnish abundant milk, but the udders and teats exhibit vesicles, "chaps," sores, etc., almost exactly like those found by Mr. Power and others in the milk diphtheria epidemics. And in the lymph of these udder vesicles the true bacillus diphtheriæ could be found. So also from the milk itself abundant colonies of the bacillus could be cultivated, whilst no other organisms could be detected. Then came a curious accidental experiment. Contrary to orders, two cats had been supplied with some of the cow's milk, and in these cats the disease called by Dr. Klein "cat diphtheria" resulted. This latter experiment is stated to be referred to by Dr. Buchanan as lacking scientific precision owing to its accidental character, but it is of much interest in connexion with the subject discussed; and will doubtless be repeated under proper precautions. Reviewing the information resulting from these experiments on cows, "we are able," said Dr. Thorne, "to appreciate the import of those apparently trivial ailments in milch cows to which so little importance has hitherto been attached by veterinarians and dairymen, but which have so commonly been discovered in association with epidemics of milk diphtheria.'

At this stage of the lectures Dr. Thorne laid down a series of propositions embodying the points which he had brought forward, and he explained that time had not permitted him to deal with all the matters which were properly included in the somewhat comprehensive title which he had originally attached to this course. Prevention of diphtheria had not been touched upon; and he had desired to deal with a number of other points, such as the stability of the contagium, the vehicles by which it could be communicated, its possible diffusion aërially, the incubative period of the disease, and its relation to croup. And, finally, he anmounced his intention of publishing the course of lectures in such form as would include these and other points which he had been compelled, by reason of time, to pass over without comment.

\section{HISTORY OF A CASE OF CEREBRAL TUMOUR :}

\section{DIAGNOSIS : OPERATION : PATHOLOGY. Read before the Glasgow Medico-Chirurgical Society.}

\section{I.-DIAGNOSIS AND LOCALISATION FROM THE NERVE SYMPTOMS.}

By T. MCCALL ANDERSON, M.D.,

Professor of Clinical Nedicine in the University of Glasgow.

Gentlames,- - I have to request your careful attention to the details of the history of this case, because it is mainly from them that the diagnosis of the seat and nature of the disease was made.

A. K. ${ }^{1}$ aged 16 , engineer's apprentice, was admitted to Ward 2 of the Western Infirmary on August 14th, 1890, complaining of fits and of paresis of the left arm and leg. No definite neurotic tendency can be traced in the family beyond the fact that his mother suffers frequently from headache, and that 1 Reported by the resident medical officer, Mr. ${ }^{-}$L. R. Sutherland, M.B., C.M. 
for a number of years he himself has been similarly affected, apparently as part of "bilious attacks." There is no history nor are there evidences of syphilis or tuberculosis. There is an indefinite history of slight discharge from the ears during infancy, but all traces of this have been absent for a number of years.

About four years and a half ago, without previous warning, and while in the act of lacing his boots, his left forearm was suddenly flexed, and, uttering a cry, he fell down in a semiinsensible condition, conscious, to a certain extent, of what was going on around him, but unable to speak or move. Ever since this fit he has complained at times of slight pain, numbness and weakness in the left hand, and within the last year or so this had been more frequent and severe. The pain and its accompanying numbness are usually experienced in the left thumb and forefinger, and, only when severe, extend to the rest of the hand and forearm.

No recurrence of fits took place till a year and a half ago, when, on May 10th, 1889, a second occurred. This was preceded by lateral oscillation of the head for about two hours. Pain and numbness were then complained of in the left thumb and forefinger, which gradually extended upwards through the left hand and forearm to the arm, finally affecting the left side of the face, including the left half of the tongue. He remembers uttering a cry and falling, and a quarter of an hour later he woke up, unconscious of what had happened, and complaining of headache and nausea. A third fit occurred on the following day, and fourteen days later a fourth These all began in much the same manner, and had much the same character and duration.

Alarmed about this state of matters, he sought admission to hospital on June 11th, 1889 , and was under treatment by mixed bromides, gr. v, increased to gr. xv t.i.d. till Angust 3rd, 1889, when he was dismissed much improved. While under observation on this first occasion he had only two fits. These were ushered in by pain in the left thumb and forefinger, extending up the arrn towards the head. The actual fit was apparently a generalised convulsion, said to have been attencied by sobbing and profuse perspiration.

A fortnight after leaving hospital the fits returned, and have since continued to recur. At times intervals of from eight to sixteen weeks elapse, at others they occur daily, even though he may be under the influence of bromides. The fits, since he left hospital, have differed from the previous ones in so far as there has been no loss of consciousness. The aura has continued as before.

For a month hefore his readmission the numbness and pain in the left hand and forearm have been becoming rapidly worse, and partial paralysis of the left arm, gradually extending to the leg, has develnped.

During the ten weeks he was under observation in hospital for the second time eighty-nine fits occurred. From August 21 st to September 17th there were no fewer than eighty-seven, on an average three daily. Under the influence of treatment they gradually became less frequent, and finally ceased. For three weeks no fits occurred. On October 10th, and again on the $26 \mathrm{th}$, a slight recurrence took place, all treatment having been suspended on the 21 st.

Examination.-The limbs of the affected side are somewhat flabby and cold. There is very decided paresis of the left arm and hand, the dynamometer registering on the right 50 kilos, on the left nil. Quite distinct, but less decided, paresis can be made out in the left leg on resisting movement. There is exaggeration of the left knee and wrist jerks, slight left ankle clonus, and the superficial reflexes are active. Tactile sensation is perfect. A feeling of numbness is experienced all over the left side, particularly in the arm and leg, and to a less extent in the left side of face and left half of the tongue, but this is not constant. There is slight facial paralysis as estimated by the usual tests. There is slight deviation of the uvula to the left, and the tongue on protrusion is slightly deflected to the affected side. Pain, at times very acute, is complained of behind and above the right ear and in the right frontal region, and, at a point 2 inches above and behind the ear, an area of distinct tenderness is discovered on pereussion.

The following are the results of the examination of the eyes, ears, and urine :-

Dr. Hinshel wood's Report on the Condition of the Eyeg
(September 11th, 1890).--"Well-marked optic neuritis present in both eyes, but all the changes are most marked in the right. The papillæ are swollen, and the normal cupping of the discs entirely obliterated. The edges of both discs are obscured, so that it is impossible to make out where the retina begins and the disc ends. The papilla are of a deep greyish-red colour, but towards the outer part are surrounded by a palish halo. The veins are dilated, and the arteries are smaller than normal. The retince, for a considerable distance round the optic discs, have lost their transparency, and have an opaque greyish appearance, which gives a very dull fundus reflex on ophthalmoscopic examination."

Dr. BarR's REPORT ON THE CONDITION OF THE EARS (October 29th, 1890).- "Right ear. Hearing power slightly under the normal ; tympanic membrane fairly normal; no perforation, cicatrix, or any evidence of present or past purulent disease. Bone conduction good. 'The tinnitus is probably connected with some form of irritation at the auditory centre in the brain. Left ear: Pear-shaped cicatrix in tympanic membrane; rest of membrane opaque. There are indications of a past purulent disease of the middle ear. Hearing power more impaired than on the right side."

Dr. W. F. SOMERVILle's RePoRT oN THE URINe (October 30th, 1890).-"A. K. ; amount of urine examined, 1.2 lite in 24 hours; colour, palish amber; odour, urinous ; reaction, neutral; specific gravity, 1022 .

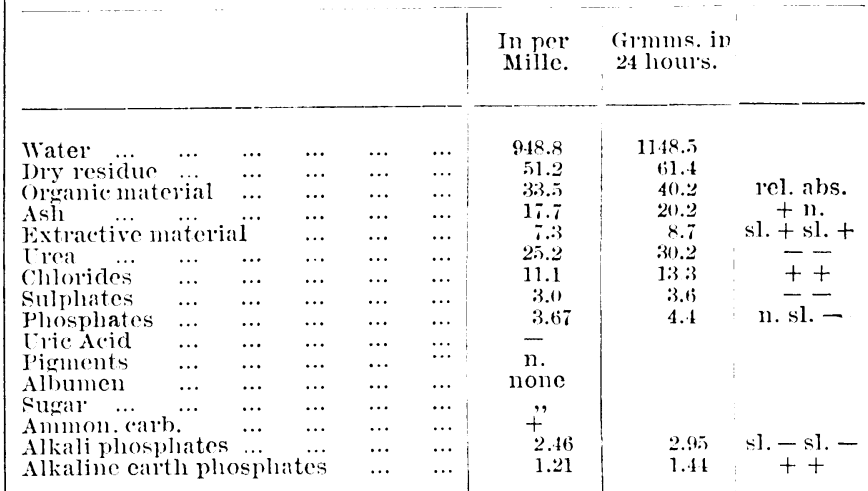

NoTe. $+=$ increased : $-=$ decreased rel. = relatively to dry residue: abs. = absolutely in twenty-four hours; sl. = slightly; $\mathrm{n.}=$ nolmal.

"Result.-I have neither seen the patient, nor do I know any clinical particulars of the case. From the examination of the urine according to the method recommended by Mr. A. E. Haswell, pathological chemist, Vienna, I find evidences of brain irritation, as shown by the increased excretion, relatively and absolutely, of the earthy phospliates, caused, perhaps, by the presence of a tumour, or by the pressure of bone on the brain-substance; or possibly, though not likely, by a very old encapsuled abscess. From the light colour of the urine, the specific gravity, the amount of urine voided in twenty-four hours, the relatively decreased urea, and the increased chlorides, the possibility of any suppurative process, as one would find in a case of abscess or of meningitis, can be quite excluded."

The following is the substance of my remarks to my clinical class prior to the operation: In reference to diagnosis, the points which we have specially to consider are two; first, the seat; and secondly, the nature of the lesion.

1. The Seat of the Lesion.-The disease is manifestly cerebral, and implicates the right side of the brain, as the resulting manifestations are almost exclusively on the left side of the body, while the paralytic phenomena indicate implication of the motor tract. But what part of the motor tract is the seat of the mischief? The symptoms point, I think, very positively to the cortex cerebri. For it must be borne in mind that in cortical lesions convulsions are very common, are frequently limited, at least at their onset, to the part whose centre is irritated, and consciousness is often retained, factors which are all present in our patient. In such cases, too, a sensory aura often precedes the epileptiform seizures, and in this instance the fits set in with numbness and pain in the left thumb and forefinger. 
This last circumstance, coupled with the fact that the paralysis began in the left arm, points to a lesion having its centre about the junction of the middle with the lower third of the ascending parietal convolution. This conclusion is somewhat supported by the pain which was complained of, and by the tenderness on percussion above and behind the right ear, although it must be remembered that the seat of pain does not necessarily correspond with the seat of the disease.

2. The Nature of the Lesion.-The history of suppurative disease of the middle ear naturally leads to the suspicion that there might be a cerebral abscess; but this suppuration occurred on the left side, and in early life, a good many years before there were any symptoms of cerebral irritation; while Dr. Barr's report furnishes evidence of prolonged absence of active disease of the middle ear. So that we may almost certainly exclude the hypothesis of abscess of the brain, more especially as Dr. Somerville's report on the urine is decidedly against such a view.

That being so, we can come to no other conclusion than that we have to deal with a tumour of some kind. The most common forms of cerebral growth are tuberculous or syphilitic in their nature, and which might possibly be influenced by the inoculation of Koch's fluid on the one hand, and antisyphilitic treatment on the other. But there is no history of a hereditary tendency to tuberculous disease, nor is there any indication of a delicacy of constitution in the patient himself And, as regards syphilis, there is no history or symptom of hereditary transmission of the taint, nor is there any suspicion of the disease having been acquired, not to speak of the age of the patient which, of itself, almost excludes it. A cancerous growth is out of the question, as the patient presents none of the characteristics of malignant disease, nor does there appear to be a family predisposition thereto.

If, then, we are right in our view that the tumour is neither tuberculous, syphilitic, nor cancerous, all that we can say further is that the intracranial growths which are most commonly met with are glioma or sarcoma ; and the only way of getting rid of them is by operative interference.

\section{II.-OPERATION FOR ITS REMOVAL : RECOVERY.}

By GEORGE BUCHANAN, M.A., M.D., LL.D.,

Professor of Clinical Surgery in the University of Glasgow.

Cerebral surgery at the present day is in much the same position as abdominal surgery was thirty years ago. In April, 1864, I performed the first successful operation of ovariotomy in Glasgow or the West of Scotland. Not very many years before that, the leading surgeon in scotland had openly declared his opinion that ovariotomy was unjustifiable, and that a surgeon who undertook that operation with a fatal result, laid himself open to a charge of culpable homicide. At the present time, owing to improved methods of operating and after-treatment, abdominal section is performed with impunity almost daily.

Cranial or cerebral surgery is now on its trial. Horsley, Macewen, Park, Bergmann, and others have clearly established that operations on the cranium for the relief of abscess and removal of morbid deposits and new growths, evidenced by certain well-ascertained nerve symptoms, can be undertaken with success. But as yet the opportunities for performing these operations have fallen into very few hands, so that it is the duty of every surgeon who can add to the limited experience to do so.

Trephining has frequently been performed in cases where there are scars on the scalp or depressions of bone indicating former injury, to which nerve symptoms, such as paralysis, convulsions, or neuralgia, etc., might be traced. A very striking example of this I published in the British Medical Journal, December 14th, 1889, p. 1318.

But the interest in the cerebral surgery of the present day is centred in those cases where the abnormal condition of the brain is diagnosed and localised by the nature and situation of the nervous affection, as first pointed out by Ferrier.

I have not for myself collected the published cases for the last two years, but the results of operations up to 1888 have been summarised in a paper by Dr. Park in the Transactions of the Congress of American Physicians and Surgeons at Washington, published in 1889 . The table includes those only in which the operation was performed according to the principles of cerebral localisation, without reference to scars or surface markings. The table contains 63 cases; the deaths were $17 ; 15$ of the cases were abscesses, subdural or subcortical ; in 11 the lesion was a tumour, exclusive of tuberculous nodules; there were 12 cysts; the other 25 were of a miscellaneous nature, such as the fibrination of a clot, gumma, tubercle, and pressure from confined and altered serous or other fluid.

These statistics, like most others, are probably defective. Additional cases may have been recorded and escaped the notice of the compiler; others may have been done and not published. No doubt every successful case has been recorded; not so with some the result of which has been negative or fatal. Still, the table is valuable, as the compilation, so far as could be achieved, of cases of the operation referred to, during the early years of its introduction.

The following remarkable case comes under the category of those included in Dr. Park's table :-

A. K., aged 17, an engineer by trade, was admitted under my care to Ward 3 of the Western Infirmary on October 30th, 1890. He had previously been in the medical wards under Professor McCall Anderson, who has given a detailed account of the history of the case and the diagnosis, which may be summed up in a few words: For three years he has been subject to epileptic attacks, which have gradually increased in frequency and severity. They always begin and sometimes are limited to the thumb and forefinger of the left hand, but frequently involve the whole limb, and sometimes the leg of the same side. Of late, the left arm has become partially paralysed, and to a slighter degree the left leg.

These symptoms seemed to a point to a gradually developing lesion, probably a tumour, in the cerebral centre presiding over the movements of the thumb, fingers, and wrist, which are very aistinctly marked out in Ferrier's map of the cerebral convolutions, at the junction of the middle and lower third of the post-Rolando convolution.

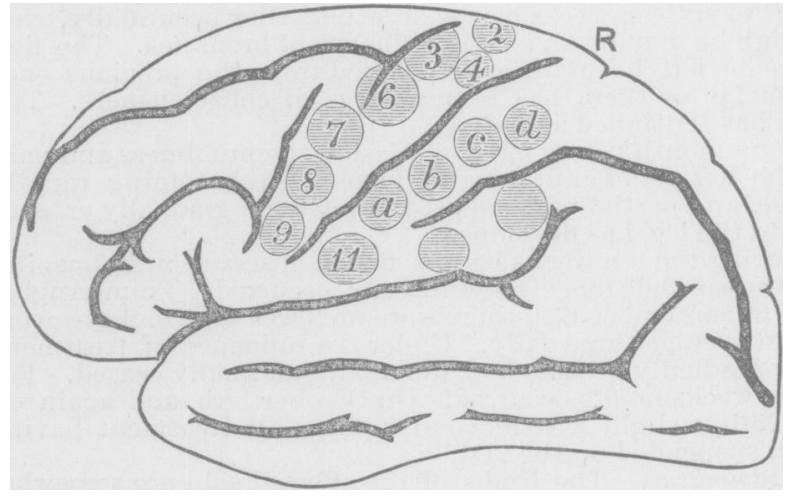

$2,3,4$, centres for the movements of arms and legs : 6, centre for supination of hand and flexion of forearm ; $a, b, c, d$, centres for hand and wrist; $11, a, b, c, d$, are on the ascending parietal convolution behind the fissure of Rolando $R$. The tumour was situated under $a$.

Accordingly, after careful consideration, Dr. Anderson and I decided that an exploratory operation should be performed on the region referred to. In the preparation of the patient, the steps of the operation, and the after-treatment, I followed rigidly the plan described by Victor Horsley, and had every reason to be satisfied with the result.

Three days before the operation the head was shaved, to allow me to study the topography of the cranium. The point at which I proposed to open the cranium was fixed on by the following measurements: the distance between the root of the nose and occipital protuberance was divided into two equal parts; half an inch behind the centre point, indicating the upper end of the fissure of Rolando, was marked. From this point a line was drawn downwards and forwards at an angle of $65^{\circ}$; this indicates the direction of Rolando's fis- 